\title{
A Novel 3D Multi-scale Lineness Filter for Vessel Detection
}

\author{
H.E. Bennink ${ }^{1}$, H.C. van Assen ${ }^{1}$, G.J. Streekstra ${ }^{2}$, R. ter Wee ${ }^{2}$, J.A.E. Spaan ${ }^{2}$, \\ and B.M. ter Haar Romeny ${ }^{1}$ \\ ${ }^{1}$ Biomedical Image Analysis, Faculty of Biomedical Engineering, \\ Eindhoven University of Technology, The Netherlands \\ 2 Department of Medical Physics, Academic Medical Center, \\ University of Amsterdam, The Netherlands
}

\begin{abstract}
The branching pattern and geometry of coronary microvessels are of high interest to understand and model the blood flow distribution and the processes of contrast invasion, ischemic changes and repair in the heart in detail. Analysis is performed on high resolution, $3 \mathrm{D}$ volumes of the arterial microvasculature of entire goat hearts, which are acquired with an imaging cryomicrotome.

Multi-scale vessel detection is an important step required for a detailed quantitative analysis of the coronary microvasculature. Based on visual inspection, the derived lineness filter shows promising results on real data and digital phantoms, on the way towards accurate computerized reconstructions of entire coronary trees.

The novel lineness filter exploits the local first and second order multiscale derivatives in order to give an intensity-independent response to line centers and to suppress unwanted responses to steep edges.
\end{abstract}

\section{Introduction}

It is necessary to improve the insight in the detailed structure of the coronary microvascular bed, as this will lead to a better understanding of e.g. the causes of local and borderline ischemia, local perfusion, and vessel remodeling. However, conventional 3D imaging does not have the required micrometer resolution.

The imaging cryomicrotome is a unique instrument that allows $3 \mathrm{D}$ imaging of large, frozen tissue samples, with a resolution of several micrometers [1. In contrast with microscopy, the imaging cryomicrotome does not image the slices, but it images the cutting surface of the remaining bulk. Because the deformation is negligible, microtomy of an entire heart yields a continuous 3D stack that contains a few billion voxels and millions of distinguishable microvessels.

Due to scattering and the large intensity differences in the imaging cryomicrotome data, it is impossible to segment the smallest vascular trees using only a simple threshold. As a result of scattering, the voxels close to, but outside large vessels are much brighter than the voxels inside the smaller vessels. The goal of the lineness filter is to enhance all line-like structure in the imaging cryomicrotome data and to suppress noise in such a way that the output can yet be segmented by a threshold [Figure 1].

N. Ayache, S. Ourselin, A. Maeder (Eds.): MICCAI 2007, Part II, LNCS 4792, pp. 436 443, 2007.

(C) Springer-Verlag Berlin Heidelberg 2007 


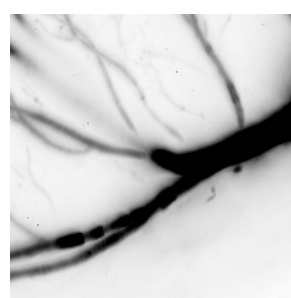

(a)

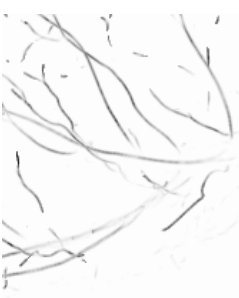

(b)

Fig. 1. (a) An inverse Maximum Intensity Projection (MIP) of 100 imaging cryomicrotome slices, showing a nutrient artery branching into smaller arterioles on the outer surface of the myocardium. The dimensions of this (sub)volume are $8 \times 8 \times 4 \mathrm{~mm}^{3}$. (b) The response of the novel lineness filter at a scale of $60 \mathrm{\mu m}$.

\section{Method}

\subsection{Local Properties of Linear Structure}

Whether structure is linear or not, depends on the scale at which it is observed. Therefore, images that contain objects of different sizes need multi-scale structure filters. Imaging cryomicrotome stacks of microvascular trees are a good example of multi-scale structure, because they contain line-like structure in a broad range of diameters.

Using the concepts of linear scale-space theory [2], differentiation of image $L(\mathbf{x})$ is defined as a convolution with derivatives of Gaussians,

$$
\frac{\partial}{\partial x} L(\mathbf{x}, \sigma)=\sigma^{\gamma} L(\mathbf{x}) * \frac{\partial}{\partial x} G(\mathbf{x}, \sigma), \quad \mathbf{x} \in \mathbb{R}^{D}, \sigma \in \mathbb{R}^{+}, \gamma \in \mathbb{R}^{0+} .
$$

Here, the $D$-dimensional Gaussian $G(\mathbf{x}, \sigma)$ is defined as

$$
G(\mathbf{x}, \sigma)=\frac{1}{\left(2 \pi \sigma^{2}\right)^{D / 2}} e^{-\frac{|\mathbf{x}|^{2}}{2 \sigma^{2}}} .
$$

The parameter $\gamma$ was introduced by Lindeberg [3] to define a family of normalized derivatives. Normalization is particularly important for a justified comparison of the response of differential operators at multiple scales. In fact, $\gamma=1$ causes the unit of the derivatives to be just 'cd' - candela, the SI base unit of luminous intensity — instead of ' $\mathrm{cd} \mathrm{m}^{-n}$ ', where $n$ denotes the order of the derivative.

Using these differential operators, the neighborhood of an image point $L(\mathbf{x})$ can be described by its local derivatives. On the center line of a line-like object, the first order derivatives are close to zero, because this point is an extremum. The second order information, however, can be used to estimate local orientation. 
Table 1. Second order structure in relation to the eigenvalues of the Hessian. ' 0 ' indicates a relatively small eigenvalue, '+' a significant positive value, and '-' a significant negative value. In the $2 \mathrm{D}$ case, the first column and second and third rows should be discarded.

\begin{tabular}{ccc|c}
$\lambda_{1}$ & $\lambda_{2}$ & $\lambda_{3}$ & Structure \\
\hline 0 & 0 & 0 & No noticeable structure \\
- & 0 & 0 & Plate-like, bright \\
+ & 0 & 0 & Plate-like, dark \\
- & - & 0 & Line-like, bright \\
+ & + & 0 & Line-like, dark \\
- & - & - & Blob-like, bright \\
+ & + & + & Blob-like, dark
\end{tabular}

This can be done via eigenvalue analysis of the Hessian matrix $(\mathbf{H})$, which is defined as the Jacobian matrix of the gradient of a function,

$$
\mathbf{H}\left[f\left(x_{1}, x_{2}, \ldots, x_{n}\right)\right]=\left(\begin{array}{cccc}
\frac{\partial^{2} f}{\partial x_{1}^{2}} & \frac{\partial^{2} f}{\partial x_{1} \partial x_{2}} & \cdots & \frac{\partial^{2} f}{\partial x_{1} \partial x_{n}} \\
\frac{\partial^{2} f}{\partial x_{2} \partial x_{1}} & \frac{\partial^{2} f}{\partial x_{2}^{2}} & \cdots & \frac{\partial^{2} f}{\partial x_{2} \partial x_{n}} \\
\vdots & \vdots & \ddots & \vdots \\
\frac{\partial^{2} f}{\partial x_{n} \partial x_{1}} & \frac{\partial^{2} f}{\partial x_{n} \partial x_{2}} & \cdots & \frac{\partial^{2} f}{\partial x_{n}^{2}}
\end{array}\right) .
$$

We will discuss the eigenvalues assuming they are sorted with respect to their absolute values:

$$
\left|\lambda_{1}\right| \geq\left|\lambda_{2}\right| \geq\left|\lambda_{3}\right|, \quad \lambda_{n} \in \mathbb{R}
$$

When $\lambda_{1}, \lambda_{2}$, and $\lambda_{3}$ share the same sign, the ratios between these eigenvalues indicate either a plate-like, line-like or blob-like structure, and their sign indicates if the structure is either brighter or darker in relation to its surroundings [45]. Table 1 summarizes the relations between $\lambda_{1}, \lambda_{2}$, and $\lambda_{3}$ for different structures. When the eigenvalues all have a relatively low absolute value, the image has no evident second order structure at the specified position in 4D scale-space.

Figure 2 uses ellipsoidal glyphs to show a geometrical interpretation of the three second order structures. The normalized eigenvectors $\left(\hat{\boldsymbol{\kappa}}_{1}, \hat{\boldsymbol{\kappa}}_{2}, \hat{\boldsymbol{\kappa}}_{3}\right)$ form an orthonormal basis that is oriented to the structure.

\subsection{Second Order Gaussian Line Filters}

As linear structure has orientation, line filters must be rotatable in order to find the best response. Using the Hessian matrix $\mathbf{H}$, the second order Gaussian derivative at image point $L(\mathbf{x})$ can be calculated in any direction in $3 \mathrm{D}$ space, given by a vector $\mathbf{v}$ :

$$
\nabla_{\mathbf{v}}^{2} L(\mathbf{x})=\hat{\mathbf{v}}^{\top} \mathbf{H}[L(\mathbf{x})] \hat{\mathbf{v}}, \quad \mathbf{v} \in \mathbb{R}^{3},
$$

where $\hat{\mathbf{v}}$ is the normalized version of $\mathbf{v}$, i.e. $\hat{\mathbf{v}}=\frac{\mathbf{v}}{|\mathbf{v}|}$, and $\nabla_{\mathbf{v}}^{2}$ denotes the directional Laplacian operator, oriented to $\mathbf{v}$. 


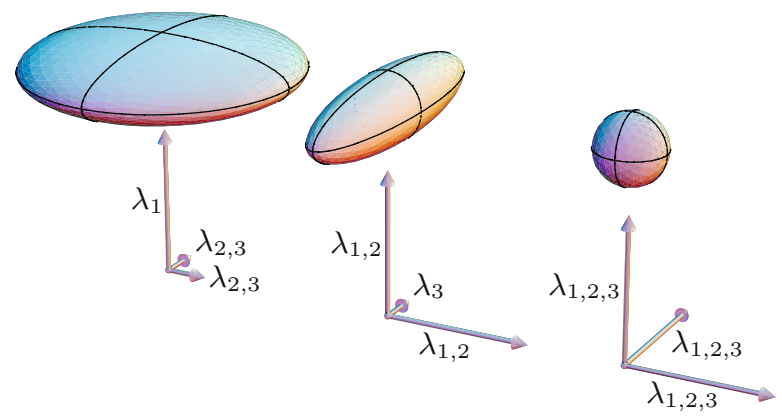

Fig. 2. Isosurface representations of plate-like, line-like, and blob-like structure, according to Table 1. The length and direction of the arrows represent respectively the eigenvalues and eigenvectors of the Hessian.

The term 'steerable filter' is frequently used to describe a class of filters of arbitrary orientation synthesized as a linear combination of a set of basis filters [6. The 2D second order Gaussian derivative in the direction of $\hat{\boldsymbol{\kappa}}_{1}$,

$$
\nabla_{\hat{\boldsymbol{\kappa}}_{1}}^{2} G(\mathbf{x}), \quad \hat{\boldsymbol{\kappa}}_{1} \in \mathbb{R}^{2}
$$

is a good 2D line filter, since the curvature will be large in that direction. Here, $\hat{\boldsymbol{\kappa}}_{1}$, is the normalized eigenvector of $\mathbf{H}$ with the largest absolute eigenvalue $\left(\lambda_{1}\right)$. The 3D equivalent of this filter involves both $\hat{\boldsymbol{\kappa}}_{1}$ and $\hat{\boldsymbol{\kappa}}_{2}$, because both vectors form a plane perpendicular to the line, while $\hat{\boldsymbol{\kappa}}_{3}$ points in the direction of the linear structure [Figure 2]:

$$
\left(\nabla_{\hat{\boldsymbol{\kappa}}_{1}}^{2}+\nabla_{\hat{\boldsymbol{\kappa}}_{2}}^{2}\right) G(\mathbf{x}), \quad \hat{\boldsymbol{\kappa}}_{n} \in \mathbb{R}^{3}
$$

Although this filter is frequently used, Jacob and Unser 7] showed that there is a better alternative without any extra cost, using Canny-like criteria [8]. The general form for a second order line filter is defined as

$$
c\left(\nabla^{2}-(\alpha+1) \nabla_{\hat{\boldsymbol{\kappa}}_{3}}^{2}\right) G(\mathbf{x}), \quad \alpha \in \mathbb{R}, c \in \mathbb{R}^{+},
$$

where $\alpha$ is the parameter to be optimized, $\hat{\boldsymbol{\kappa}}_{3}$ is the feature orientation, and $c$ is a normalization factor. It turns out that $\alpha=\frac{2}{3}$ optimizes the criteria. Note that $\alpha=0$ gives the 'default' filter as in (7).

\subsection{A Novel Lineness Filter}

Using Gaussian derivatives and the knowledge of the Hessian eigenvalues and the Canny-optimized filter, a novel lineness filter is constructed. This filter is intensity independent and suppresses the undesired response to strong edges as well - two issues where other line filters often fail, but that are particularly important for smooth enhancement and segmentation of line centers. Table 2 gives an overview of the three basic components of this filter. 
Table 2. Components of the novel lineness filter. Because of the normalization factor $\sigma^{\gamma}$ in (11), the unit of these measures depends on the value of $\gamma$.

\begin{tabular}{c|c|l} 
Measure & Unit $(\gamma=\mathbf{1})$ & Description \\
\hline$\frac{\left|\lambda_{2}\right|}{\left|\lambda_{1}\right|}$ & 1 & $\begin{array}{l}\text { Deviation from plate-like structure, equals 0 in } \\
\text { case of a perfect plate [Figure 2]. } \\
\text { Response to the Canny-optimized second order } \\
\text { line filter (8) })\end{array}$ \\
$|\nabla L(\mathbf{x})|$ & cd & $\begin{array}{l}\text { Gradient magnitude, equals 0 in mathematical } \\
\text { extrema, thus in the center of the structure. }\end{array}$
\end{tabular}

Note that some of the measures in this table have the unit 'cd', i.e. these measures are dependent on the signal intensity. This dependency is undesired, because bright linear structure should not be considered 'more linear' than less bright linear structure. This problem can be overcome in a simple fashion by division by $L(\mathbf{x})$, which requires a low threshold to prevent division by nearlyzero background values. In modalities like MRA or CTA, any intensity bias should be corrected beforehand.

The gradient magnitude, $|\boldsymbol{\nabla} L(\mathbf{x})|$, is hardly used in common line filters, although it is a fundamental property of structural centers that the gradient vanishes This property appears to be very effective regarding the suppression of undesired responses that most line filters have to strong edges.

According to the components in table 2, 'lineness' is defined as

$$
\mathcal{L}(\mathbf{x})=\left(\frac{\lambda_{2}^{2}}{\lambda_{1}^{2}}\right)^{c_{1}} \frac{1}{L(\mathbf{x})}\left(\left(\nabla^{2}-\frac{5}{3} \nabla_{\hat{\boldsymbol{\kappa}}_{3}}^{2}\right) L(\mathbf{x})-c_{2}|\nabla L(\mathbf{x})|\right)
$$

where $c_{1} \in \mathbb{R}^{0+}$ denotes the sensitivity to the roundness of line profiles and $c_{2} \in \mathbb{R}^{0+}$ is the edge suppression factor. $c_{1}=1$ and $c_{2}=1.5$ give satisfying results on the imaging cryomicrotome data. The multi-scale response is given by the maximum response over a certain range of scales, and because of the normalization factor $\sigma^{\gamma}$ (1), this maximal response is given at a scale $\sigma$ that is $\sqrt{2}$ times the vessel radius, assuming the vessel has a Gaussian profile [3].

\section{Results}

\subsection{Results on Digital Line Phantoms}

In order to compare the lineness filter $\mathcal{L}(\mathbf{x})$ to other filters, four general types of phantoms are made that deal with the following common issues;

- additive, Gaussian distributed noise (type N);

- lines of a different scale for which the intensities differ (type S);

- curved lines (type C);

- bifurcations (type B). 


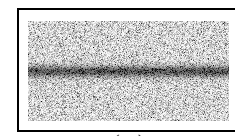

(a)

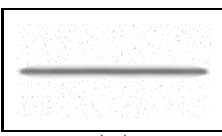

(b)

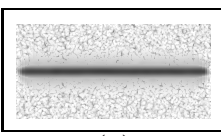

(c)

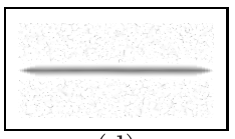

(d)

Fig. 3. (a) The medial slice of phantom N-64-G, containing a line with a Gaussian profile and noise with a standard deviation of 64. (b), (c), and (d) show the multiscale responses of respectively Frangi's filter, the Canny-optimized filter, and the novel lineness filter to N-64-G $(1 \leq \sigma \leq 8$ pixels).

Because filter responses are also dependent on the line profile, both, a Gaussian profile (type G) and a box profile (type B) are used. Note that the profiles of the vessels in the imaging cryomicrotome data are neither Gaussian, nor boxed, but somewhat in between. Besides the two line profiles, each of the phantoms shows four variations in scale or intensity, resulting in a total of 32 line phantoms.

All phantoms are filtered on four subsequent scales with the vessel-likeliness function introduced by Frangi [4] (type F), the Canny-optimized second order line filter [7] (type C), and the novel lineness filter (type L). The parameters for Frangi's vessel-likeliness function are $\alpha=0.5, \beta=0.5$, and $c=32$ (the line intensity is 128), except for the type $\mathrm{S}$ phantom, where $c$ is set to a quarter of the intensity of the thin line. The parameters for $\mathcal{L}(\mathbf{x})$ are set to $c_{1}=1$ and $c_{2}=1.5$, and all of the filters are subject to a threshold of 12 .

The type N phantoms, with Gaussian distributed noise, show that Frangi's filter and $\mathcal{L}(\mathbf{x})$ are quite insensitive to this kind of noise, while the Cannyoptimized filter shows a significantly higher response to the noise [Figure 3 ].

Apart from the fact that Frangi's vessel-likeliness function and the Cannyoptimized filter are intensity dependent, all filters perform quite comparably on the type $\mathrm{S}$ phantoms, on the condition that the line profile is Gaussian. Although, when the line profile shows steeper gradients, as in the box profile [Figure 4a], Frangi's filter (type F) and the Canny-optimized filter (type C) both give undesired responses to the edges of thick lines [Figure $4 \mathrm{~b}$ and $\mathrm{c}] . \mathcal{L}(\mathbf{x})$ however, only responds to the center line [Figure $4 \mathrm{~d}$ and e].

Curved lines that have a sinusoidal or spiraling twist are a difficult case in multi-scale line filtering. Like a rope or a DNA-strand, these objects appear as a twisted line on a small scale, but as a more or less straight line on a larger scale. This is a 'problem' of all multi-scale line filters, which is illustrated in Figure 5.

Although bifurcations are part of networks of linear structure, a bifurcation is not linear by itself. Eigenvalue analysis of the Hessian matrix [Table 1] would indicate rather blob-like or plate-like structure instead of like-like structure, i.e. true line filters should not respond to bifurcations. Type B phantoms [Figure 6] confirm that the response of all line filters to a bifurcation is weak.

\subsection{Results on Imaging Cryomicrotome Data}

Figure 7 shows a comparison of Frangi's vessel-likeliness function, the Cannyoptimized second order line filter, and the lineness filter on a scale of $60 \mu \mathrm{m}$. 


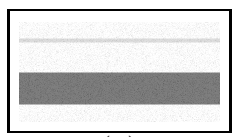

(a)

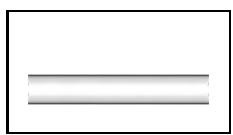

(b)

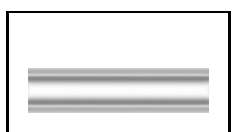

(c)

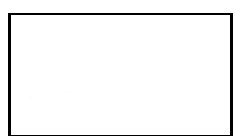

(d)

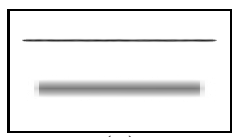

(e)

Fig. 4. (a) The medial slice of phantom S-32-B, containing a thin and a 16 pixel thick line, both having a box profile (type B). (b), (c), and (d) show the responses of respectively Frangi's filter, the Canny-optimized filter, and the novel lineness filter to S$32-\mathrm{B}$ at $\sigma=4$ pixels. (e) The multi-scale response of $\mathcal{L}(\mathbf{x})$ to S-32-B ( $1 \leq \sigma \leq 8$ pixels). Note that the novel lineness filter does not respond at all at $\sigma=4$ pixels.

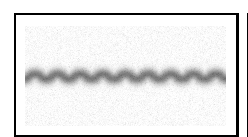

(a)

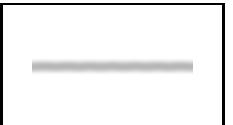

(b)

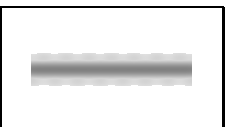

(c)

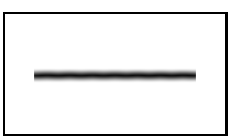

(d)

Fig. 5. (a) Medial slice of phantom C-4-G, showing a curved line with a radius of 4 pixels and Gaussian profile. (b), (c), and (d) show the responses of respectively Frangi's filter, the Canny-optimized filter, and the novel lineness filter at a scale of $\sigma=8$ pixels. The curves of the phantom have almost disappeared on this scale.

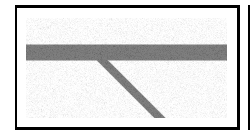

(a)

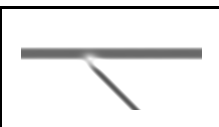

(b)

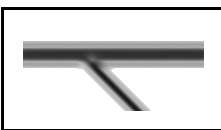

(c)

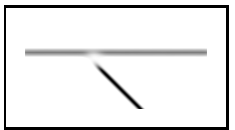

(d)

Fig. 6. (a) Medial slice of phantom B-4-B, a bifurcation of lines with radii of 8 and 4 pixels, both having a box profile. (b), (c), and (d) responses of respectively Frangi's filter, the Canny-optimized filter, and the novel lineness filter at a scale of $\sigma=4$ pixels.

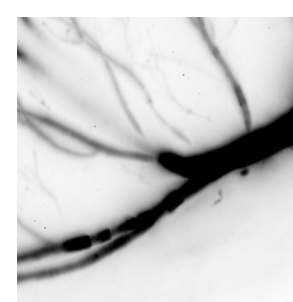

(a)

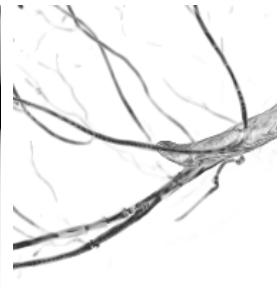

(b)

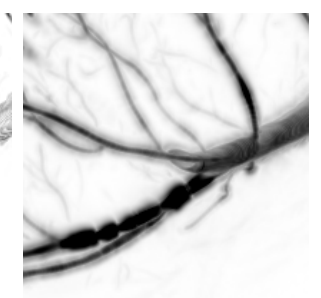

(c)

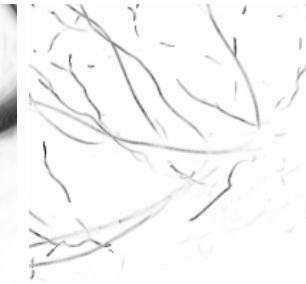

(d)

Fig. 7. (a) The volume shown in Figure 1 1. (b), (c), and (d) show inverse MIPs of the responses of respectively Frangi's vessel-likeliness function, the Canny-optimized second order line filter, and the lineness filter on a scale of $60 \mu \mathrm{m}$. The parameters for the Frangi's filter are $\alpha=0.5, \beta=0.5$, and $c=25$. The parameters for the lineness filter (9) are $c_{1}=1$ and $c_{2}=1.5$, and its threshold is set to $L(\mathbf{x})>12$ (the maximum intensity is 255). Note that in (d) some vessels appear discontinuous because of the threshold or because their medial axis moves out of the imaged volume. 
Especially the undesired responses to large, bright vessels on smaller scales are significantly reduced using the novel lineness filter; note that both Frangi's filter and the Canny-optimized filter give a high response to the large artery. Due to the threshold, however, some of the smaller vessels became discontinuous.

\section{Discussion}

The novel lineness filter, $\mathcal{L}(\mathbf{x})$, proves to get the best out of the cryomicrotome data. Because the values $c_{1}=1$ and $c_{2}=1.5$ are obtained more or less empirically, these could possibly be optimized to yield even better results.

A drawback of $\mathcal{L}(\mathbf{x})$ that can be seen in the phantoms as well as in most of real images are the weak responses to bifurcations, which result in disconnected branches. However, this phenomenon is a 'problem' that occurs in any line filter. A high order filter or postprocessing step is required to correct for this problem.

A second drawback of the filter is its multi-scale response to twisted lines, as shown in subsection 3.1. This type of structure is not very rare in microvascular images, because vessels in areas of angiogenesis show similar behavior. A solution to this (potential) problem may be found in the field of scale-space analysis. For example, edges and other extrema, such as line centers, can be traced down to smaller scales using scale-space signatures, as described in 2 .

An implementation in $\mathrm{C}++$ makes this method applicable in practice, even for the complete $2000^{3}$-voxel volumes used here. Overall, the derived filter shows promising results on the way to accurate computerized reconstructions of entire coronary trees. Finally, accurate diameter measurements are necessary to complete the quantitative reconstruction of coronary arterial trees.

\section{References}

1. Spaan, J.A.E., ter Wee, R., van Teeffelen, J.W.G.E., Streekstra, G., Siebes, M., Kolyva, C., Vink, H., Fokkema, D.S., VanBavel, E.: Visualization of intramural coronary vasculature by an imaging cryomicrotome suggests compartmentalisation of myocardial perfusion areas. Med. Biol. Eng. Comput. 43(4), 431-435 (2005)

2. ter Haar Romeny, B.M.: Front-End Vision and Multi-Scale Image Analysis: MultiScale Computer Vision Theory and Applications, written in Mathematica, 1st edn., vol. 27. Kluwer Academic Publishers, Boston, MA (2003)

3. Lindeberg, T.: Edge detection and ridge detection with automatic scale selection. Int. J. Comput. Vision 30(2), 77-116 (1996)

4. Frangi, A.F.: Three-Dimensional Model-Based Analysis of Vascular and Cardiac Images. PhD thesis, University of Utrecht (2001)

5. Sato, Y., Tamura, S.: Detection and quantification of line and sheet structures in 3-D images. In: Delp, S.L., DiGoia, A.M., Jaramaz, B. (eds.) MICCAI 2000. LNCS, vol. 1935, pp. 154-165. Springer, Heidelberg (2000)

6. Freeman, W.T., Adelson, H.A.: The design and use of steerable filters. IEEE T Pattern Anal. 13(9), 891-906 (1991)

7. Jacob, M., Unser, M.: Design of steerable filters for feature detection using Cannylike criteria. IEEE T Pattern Anal. 26(8), 1007-1019 (2004)

8. Canny, J.: A computational approach to edge detection. IEEE T Pattern Anal. 8(6), 679-698 (1986) 